\title{
Persistent fever in an infant: incomplete Kawasaki disease
}

\author{
Kevin C. Harris MD MHSc, Martin C.K. Hosking MD
}

A four-month-old white girl presented to her local hospital when her parents noticed that her left upper extremity had turned blue from her midforearm to her fingertips. When she was nine weeks old, a persistent fever had developed associated with a patchy macular rash extending over her trunk and extremities. The fever had responded transiently to antipyretics but recurred and persisted for two weeks, when both the fever and rash resolved. Still, she did not recover clinically, and her parents found her fussy and inconsolable. In addition, her parents were concerned about her red, cracked lips. She was transported from the local hospital to our pediatric intensive care unit, where our observation unit is located.

On admission, the patient was afebrile and extremely fussy with stable hemodynamics (heart rate was 160 beats/min, levels of oxygen saturation ranged from $92 \%$ to $94 \%$ on room air and systemic blood pressure was $86 / 42 \mathrm{~mm} \mathrm{Hg}$ ). She had diminished brachial and radial pulses in her left arm with easily palpable right brachial, radial and femoral pulses. On her left hand, her distal fingers were black (Figure 1). Her lips were erythematous and cracked, but there were no features of conjunctivitis, lymphadenopathy or rash. Auscultation of the lungs was clear. Her precordium was active with normal heart sounds, a soft gallop and a grade III of VI regurgitant murmur at the apex radiating to the axilla. Her liver edge was palpable $2 \mathrm{~cm}$ below the costal margin.

Results of laboratory investigations showed marked elevation of her inflammatory markers (C-reactive protein level was 176 [normal < 5] $\mathrm{mg} / \mathrm{L}$, erythrocyte sedimentation rate was 46 [normal 0-30] $\mathrm{mm} / \mathrm{h}$ ). She was anemic (hemoglobin level was 82 [normal 95-135] g/L) with an elevated leukocyte count of 36.9 (normal 5.0 $17.5) \times 10^{9} / \mathrm{L}$ and hypoalbuminemia (albumin level was 28 [normal 34-42] g/L). She had sterile pyuria. Serum transaminase levels and platelet count were normal. A workup for infection was negative. A chest radiograph showed cardiomegaly with increased pulmonary vascular

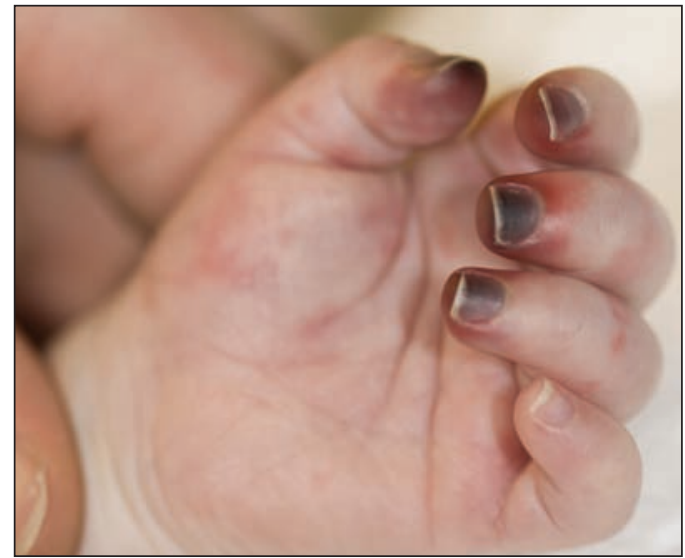

Competing interests: None declared.

This article has been peer reviewed.

Correspondence to:

Dr. Kevin C. Harris,

kharris2@cw.bc.ca

CMAJ 2011. DOI:10.1503 /cmaj.110032

Figure 1: Left hand of a four-month-old girl showing ischemia of the fingertips on digits 1 through 4.

markings. A 12-lead electrocardiogram showed biventricular hypertrophy with no features suggestive of ischemia. An echocardiogram showed mitral regurgitation, a small pericardial effusion and giant aneurysms of the coronary artery. Left ventricular function was preserved, with normal ejection and shortening fractions and no obvious segmental dyskinesis.

Based on our patient's history of prolonged fever, rash, erythematous lips, signs of systemic inflammation and coronary aneurysms, we diagnosed incomplete Kawasaki disease. We started treatment with intravenous immunoglobulin, along with a three-day course of pulse methylprednisolone as rescue therapy, recognizing that she was already in the subacute phase of her illness.

\section{KEY POINTS}

- Kawasaki disease should be considered in young children with prolonged fever (five days or more).

- Infants with Kawasaki disease often present with fever but may not have other classic signs.

- Children with Kawasaki disease who are less than one year or more than nine years of age, male or of Asian ancestry have the highest risk for coronary aneurysm.

- The cardiac sequelae of coronary aneurysms are often preventable if the diagnosis of Kawasaki disease is made and treatment with intravenous immunoglobulin is started within 10 days of the onset of symptoms. 
At cardiac catheterization, end-diastolic pressure in her left ventricle was normal $(8 \mathrm{~mm} \mathrm{Hg})$. Angiography showed extensive systemic vasculitis, with multiple aneurysms and areas of local stenosis of the central, peripheral and coronary vasculature. The left subclavian angiogram showed a complete occlusion of the left axillary artery with collateral arterial vessels supplying the brachial artery. An aortic root injection showed complete occlusion of the right coronary artery (Figure 2A) with extensive ectasia and aneurysm of the left main and left anterior descending and circumflex arteries. Thoracic and abdominal angiography showed diffuse involve-

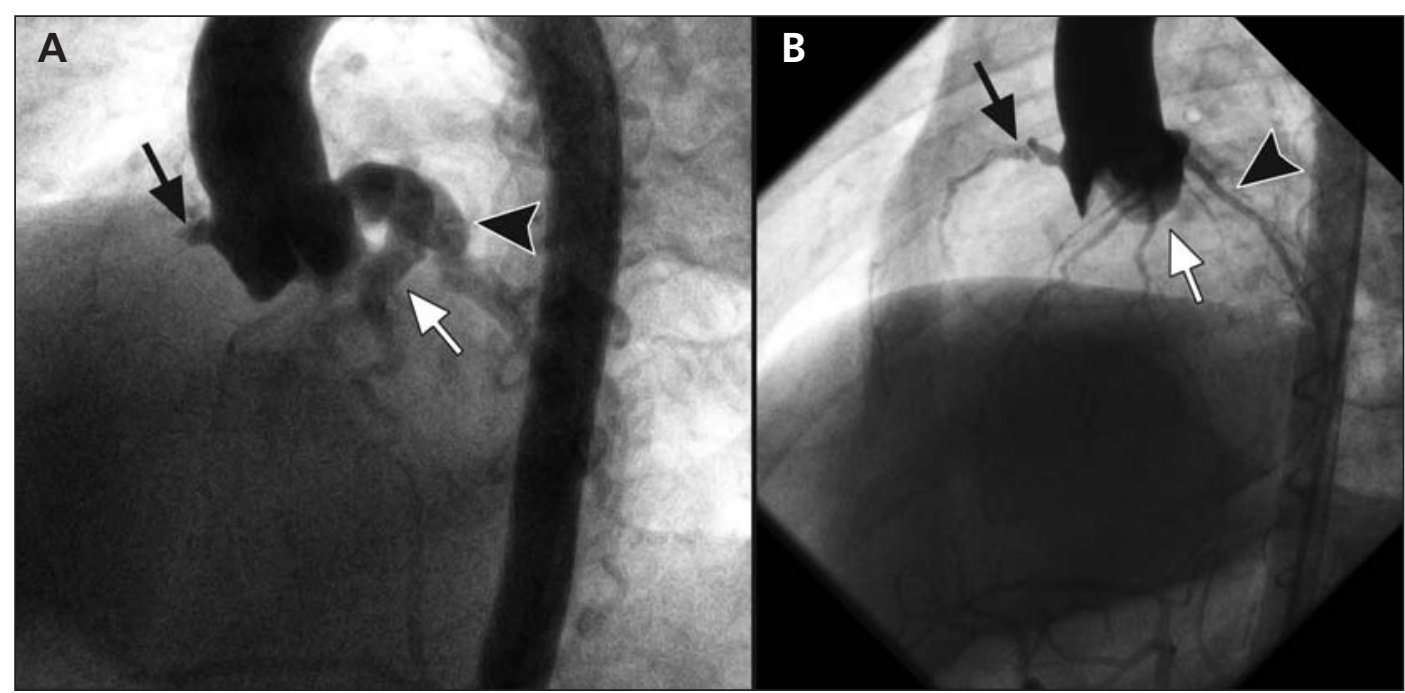

Figure 2: (A) Aortic root angiogram (left anterior oblique projection) showing aneurysmal dilatation extending from the left main coronary artery down the left anterior descending (white arrow) and circumflex (arrowhead) arteries. There is complete occlusion of the right coronary artery (black arrow). (B) Angiogram done one year later showing recovery of the right coronary artery (black arrow) and highgrade proximal stenosis. The left main, left anterior descending (white arrow) and circumflex (arrowhead) arteries now show minimal residual dilatation.

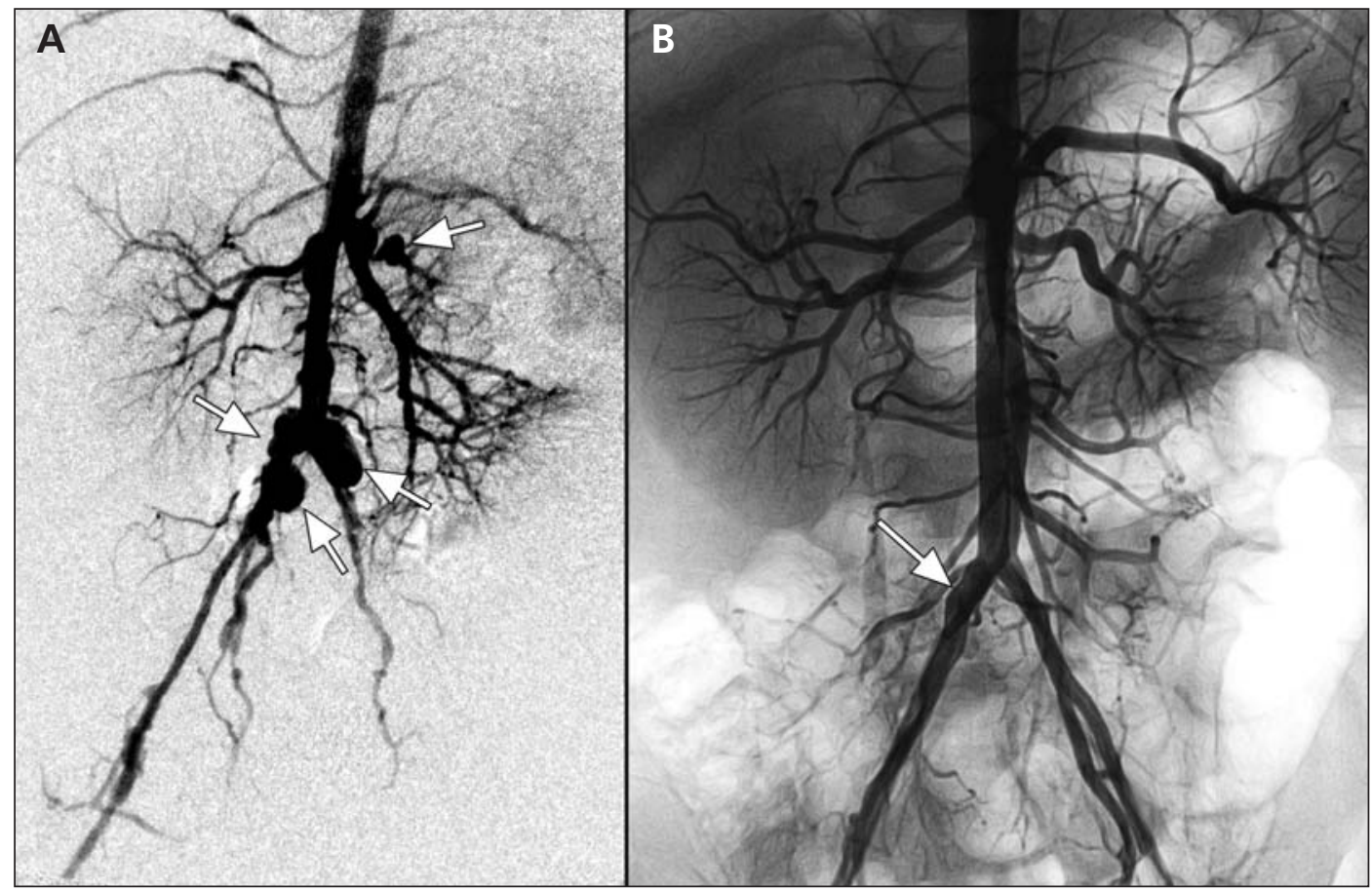

Figure 3: (A) Abdominal angiogram (arterial phase, anterior posterior view) showing extensive vascular involvement of the distal abdominal aorta, renal arteries, celiac axis and superior mesenteric artery, and bifurcation of the aorta into the iliac arteries (arrows). (B) Angiogram done one year later showing normal calibre of the renal arteries, celiac axis, superior and inferior mesenteric vascular bed and abdominal aorta. Persistent ectasia of the right common iliac artery (arrow) is shown. 
ment of the mesenteric, renal and iliac arteries (Figure 3A). During angiography, we undertook balloon dilatation of the patient's left axillary artery, and we injected tissue plasminogen activator in an attempt to improve perfusion to her left arm. This resulted in a transient improvement of flow; however, the vessel underwent rapid thrombosis and reocclusion.

In light of extensive vascular involvement, we decided to use infliximab (inhibitor of tumour necrosis factor $\alpha$ ) and abciximab (inhibitor of platelet glycoprotein IIb/IIIa receptors), along with a tapering course of steroids. The patient's condition improved; her inflammatory markers rapidly returned to normal and her fussiness resolved. She was given digoxin, furosemide and captopril for her clinical heart failure, as well as antiplatelet and anticoagulant therapy. We administered nitropaste to her fingers during her four-week stay in hospital.

Six months later, the terminal aspects of the patient's distal fingers autoamputated. Follow-up angiography a year after her initial presentation showed recanalized right coronary (Figure 2B) and axillary arteries, with marked improvement of the systemic vasculopathy (Figure 3B). At 22 months of age, she remained clinically asymptomatic with optimal weight gain and normal indices of cardiac function.

\section{Discussion}

The most common cause of acquired heart disease in children in Canada, Kawasaki disease is an autoimmune condition that affects the blood vessels, skin, lymph nodes and mucous membranes. In the United States, its incidence is 1 in 10000 children in the general population. ${ }^{1}$ Kawasaki disease is more common in boys (ratio 1.5:1.0) and children of Asian ancestry. More than $75 \%$ of children who present with Kawasaki disease are younger than five years. ${ }^{1} \mathrm{~A}$ recent nationwide survey in Japan showed the risk is as high as 17 in 10000 for Japanese children. ${ }^{2}$ The diagnosis is established based on clinical criteria (Box 1). The syndrome leads to a generalized vasculitis with a tendency for formation of aneurysms in small and medium-sized vessels, particularly the coronary arteries. If the syndrome is left untreated, coronary aneurysms occur in $15 \%-25 \%$ of affected children. ${ }^{1}$

\section{Risk factors for aneurysm}

In our experience, the vast majority of children who have giant aneurysms at presentation during the acute phase of Kawasaki disease are less than one year of age. Because this group often presents with incomplete diagnostic criteria, they have a particularly high risk for delayed or missed diagnosis. Children who are older than nine years, male or of Asian ancestry also have an elevated risk for aneurysm. ${ }^{3}$

A prospective cohort study involving children with Kawasaki disease examined serial measurements taken of the children's coronary arteries as the dimensions returned to normal. The study showed that delay of appropriate therapy with intravenous immunoglobulin, lower serum immunoglobulin levels at baseline and lower minimum serum albumin levels are independent risk factors for coronary artery damage. ${ }^{4}$ Prospective randomized controlled trials have clearly shown that intravenous immunoglobulin can reduce the risk of coronary aneurysm substantially to about $1 \%-5 \%$.

\section{Incomplete (atypical) Kawasaki disease}

Children who do not fulfill all the diagnostic criteria for Kawasaki disease but have persistent symptoms of fever warrant special attention. The American Heart Association developed an algorithm to assist in the diagnosis of Kawasaki disease in these challenging presentations (Figure 4) ${ }^{1,3} \mathrm{~A}$ recent retrospective review of presentations of Kawasaki disease with coronary aneurysms showed that $30 \%$ of children had incomplete criteria and that $19 \%$ were less than six months of age. ${ }^{5}$ In their analysis, the authors found that application of the algorithm by the American Heart Association would have identified and given appropriate treatment for $97 \%$ of children who developed coronary aneurysms, whereas only $70 \%$ of those children would have been identified and received appropriate treatment using the full criteria for Kawasaki disease.

Risk factors for incomplete Kawasaki disease are not well established. However, in our experience, boys and children of Asian ancestry appear to have the highest risk, which is consistent with the risk factors for the complete syndrome.

\section{Box 1: Simplified criteria* for diagnosis of} Kawasaki disease ${ }^{1}$

- Fever for five days or longer, with at least four of the following five signs:

- Rash

- Cervical lymphadenopathy

- Edema or erythema of the hands or feet (peeling later in clinical course)

- Erythema of the lips or the oral mucosa

- Bilateral nonpurulent conjunctivitis

- Exclusion of other diseases with similar findings.

*Full criteria is available in Newburger et al. ${ }^{1}$ 
Evaluation of suspected incomplete Kawasaki disease ${ }^{a}$

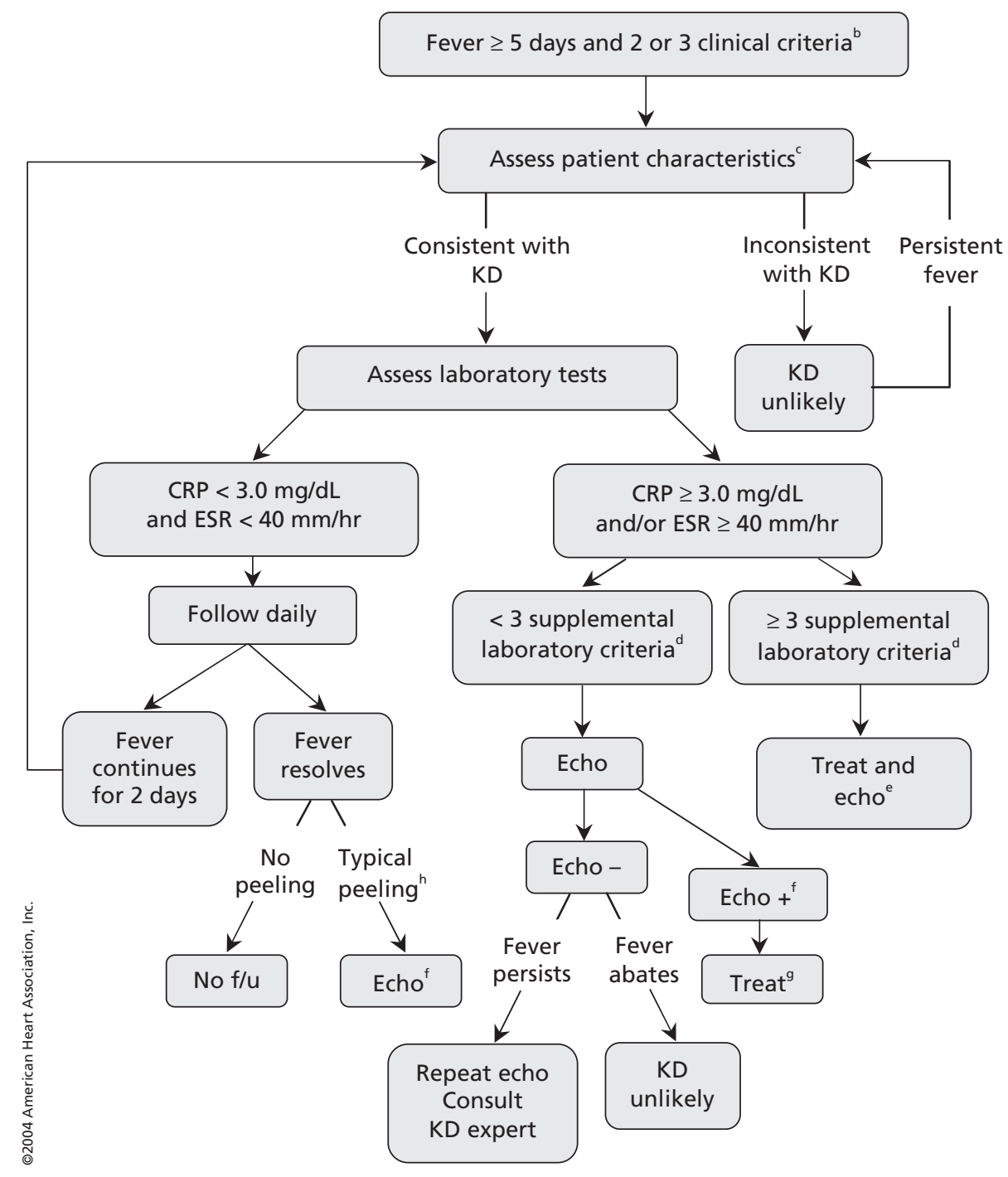

Figure 4: Evaluation of suspected incomplete Kawasaki disease. (a) In the absence of a gold standard for diagnosis, this algorithm cannot be evidence-based but rather represents the informed opinion of the expert committee. Consultation with an expert should be sought any time assistance is needed. (b) Infants six months of age or younger on day seven or later of fever without other explanation should undergo laboratory testing and, if evidence of systemic inflammation is found, an echocardiogram, even if the infants have no clinical criteria. (c) Patient characteristics suggesting Kawasaki disease are listed in Box 1. Characteristics suggesting disease other than Kawasaki disease include exudative conjunctivitis, exudative pharyngitis, discrete intraoral lesions, bullous or vesicular rash or generalized adenopathy. Consider alternative diagnoses. (d) Supplemental laboratory criteria include albumin $\leq 3.0 \mathrm{~g} / \mathrm{dL}[\leq 30 \mathrm{~g} / \mathrm{L}]$, anemia for age, elevation of alanine aminotransferase, platelets after seven days $\geq 450000 / \mathrm{mm}^{3}$, white blood cell count $\geq 15000 / \mathrm{mm}^{3}$ and urine $\geq 10$ white blood cells/high-power field. (e) Can treat before performing echocardiogram. (f) Echocardiogram is considered positive for purposes of this algorithm if any of three conditions are met: $z$ score of left anterior descending coronary artery or right coronary artery $\geq 2.5$, coronary arteries meet Japanese Ministry of Health criteria for aneurysms, or three or more other suggestive features exist, including perivascular brightness, lack of tapering, decreased left ventricular function, mitral regurgitation, pericardial effusion, or $z$ scores in left anterior descending coronary artery or right coronary artery of 2-2.5. (g) If the echocardiogram is positive, treatment should be given to children within 10 days of fever onset and those beyond day 10 with clinical and laboratory signs (CRP, ESR) of ongoing inflammation. (h) Typical peeling begins under nail bed of fingers and then toes. ${ }^{3}$ Reprinted, with permission, from Newburger et al ${ }^{1}$ Circulation. 2004;110:27472771 @2004 American Heart Association, Inc. http://circ.ahajournals.org/cgi/content/full/110/17/2747.

Note: CRP = C-reactive protein, echo = echocardiogram, ESR = erythrocyte sedimentation rate, f/u = follow-up, $\mathrm{KD}=$ Kawasaki disease.

[Editor's. note: the unit of measure for CRP levels in the figure is $\mathrm{mg} / \mathrm{dL}$.] 
Young children who present with fever, rash and fussiness may be suspected to have other conditions such as adenovirus, enterovirus, scarlet fever or hypersensitivity to drugs (particularly a reaction to antibiotics recently prescribed for fever with or without lymphadenopathy). ${ }^{1}$

Of particular relevance to this presentation, the current guidelines recommend that infants aged six months or younger with fever for seven days or longer without other explanation should undergo laboratory investigation for indicators of a systemic vascular response (i.e., C-reactive protein level, erythrocyte sedimentation rate). In presentations with evidence of systemic inflammation, echocardiography should be performed even if there are no clinical criteria of Kawasaki disease. ${ }^{1}$

There are several reports of infantile Kawasaki disease in the literature, including sporadic instances of peripheral gangrene. However, the extent of vascular involvement in our patient is striking, and, despite improvement of her extensive systemic vasculopathy, she is left with highgrade stenosis of the proximal right coronary artery. Her positive response to therapy contrasts with the natural history of giant coronary aneurysms, which tends to involve obstruction or stenosis. ${ }^{6}$

Although the classic presentation meeting all criteria for Kawasaki disease makes for a straightforward diagnosis, clinicians may wish to keep incomplete forms of the illness in mind when assessing high-risk infants who do not recover from febrile illnesses as expected.

\section{References}

1. Newburger JW, Takahashi M, Gerber MA, et al. Diagnosis, treatment, and long-term management of Kawasaki disease. Circulation 2004;110:2747-71.

2. Nakamura Y, Yashiro M, Uehara R, et al. Increasing incidence of Kawasaki disease in Japan: the nationwide survey. Pediatr Int 2008;50:287-90.

3. Belay ED, Maddox RA, Holman RC, et al. Kawasaki syndrome and risk factors for coronary artery abnormalities: United States, 1994-2003. Pediatr Infect Dis J 2006;25:245-9.

4. McCrindle BW, Li JS, Minich LL, et al. Coronary artery involvement in children with Kawasaki disease. Circulation 2007;116:174-9.

5. Yellen ES, Gauvreau K, Takahashi M, et al. Performance of 2004 American Heart Association recommendations for treatment of Kawasaki disease. Pediatrics 2010;125:e234-41.

6. Akagi T, Rose V, Benson LN, et al. Outcome of coronary artery aneurysms after Kawasaki disease. J Pediatr 1992;121:689-94.

Affiliations: From the Children's Heart Centre, BC Children's Hospital, Vancouver, BC

Contributors: Both authors were responsible for the conception of the manuscript. Kevin C. Harris drafted the manuscript, which both authors revised. Both authors have approved the final version submitted for publication.

Acknowledgements: The authors thank the patient and the patient's family for consenting to publication of the case, photograph and angiograms. The authors thank Dr. Jim Potts for his assistance with formatting the images.

\section{Helpful resources}

- Guidelines of the American Heart Association: Diagnosis, treatment, and long-term management of Kawasaki disease. http://circ.ahajournals.org/cgi/content/full/110 $/ 17 / 2747$

- Information for parents from the Kawasaki Disease Foundation: www.kdfoundation.org /forms/kdfbrochure.pdf 